\title{
A comprehensive logic-based model of the human immune system to study the dynamics responses to mono- and coinfections
}

Bhanwar Lal Puniya ${ }^{1 \mathbb{P}}$, Robert Moore ${ }^{1 \mathbb{P}}$, Akram Mohammed ${ }^{1}$, Rada Amin ${ }^{1}$, Alyssa La Fleur², Tomáš Helikar ${ }^{1 *}$

${ }^{1}$ Department of Biochemistry, University of Nebraska-Lincoln, Lincoln, Nebraska, USA

${ }^{2}$ Department of Mathematics and Computer Science, Whitworth University.

${ }^{\mathbb{P}}$ These authors have equally contributed to this work

*To whom correspondence should be addressed.

\section{Contact information:}

Tomáš Helikar, Ph.D., Associate Professor, Department of Biochemistry, University of Nebraska-Lincoln, USA, Email: thelikar2@unl.edu

\begin{abstract}
The human immune system, which protects against pathogens and diseases, is a complex network of cells and molecules. The effects of complex dynamical interactions of pathogens and immune cells on the immune response can be studied using computational models. However, a model of the entire immune system is still lacking. Here, we developed a comprehensive computational model that integrates innate and adaptive immune cells, cytokines, immunoglobulins, and nine common pathogens from different classes of virus, bacteria, parasites, and fungi. This model was used to investigate the dynamics of the immune system under two scenarios: (1) single infection with pathogens, and (2) various medically relevant pathogen coinfections. In coinfections, we found that the order of infecting pathogens has a significant impact on the dynamics of cytokines and immunoglobulins. Thus, our model provides a tool to simulate immune responses under different dosage of pathogens and their combinations, which can be further extended and used as a tool for drug discovery and immunotherapy. Furthermore, the model provides a comprehensive and simulatable blueprint of the human immune system as a result of the synthesis of the vast knowledge about the network-like interactions of various components of the system.
\end{abstract}

\section{Introduction}

The human immune system is a complex and dynamic network of immune cells and molecules that detect and destroy pathogens and abnormal cells to fight diseases. The types of immune responses vary according to the type of infecting pathogens (1-5). For example, interferon- $\gamma$-secreting $\mathrm{T}$ helper 1 (Th1) cells are effective against intracellular bacteria and viruses; interleukin-4-secreting T helper 2 (Th2) cells target parasitic pathogens; interleukin-17-secreting T helper 17 (Th17) cells protect against extracellular bacteria (5). 
Furthermore, certain types of cytokines coexisting at different concentrations in the environment can stimulate CD4+ T cells to develop into phenotypes with mixed characteristics $(6,7)$. Similarly, understanding the immune response becomes more complicated when multiple pathogens interact with the immune system simultaneously or one after another. Studied examples of such coinfections include HIV and tuberculosis (8), tuberculosis and influenza (9), and malaria and influenza (10).

To understand such complex dynamics of the immune response against multiple infections requires dynamic, systems-level studies. Systems-level approaches have been used at the population and single-cell level to identify gene signatures associated with diseases $(11,12)$, to elucidate critical signaling pathways, and to identify communication between cells and molecules in response to infections and vaccines (13). However, capturing the large numbers of interacting cells in various states, and diverse scales of immune responses, as a whole, is still challenging through experiments.

Computational models enable a systems-level analysis of the immune system's dynamics. The dynamics of small to large systems have been explored using logical (6,14-16), flux-based $(17,18)$, and ordinary or partial differential equation-based modeling techniques $(19,20)$. For instance, a mathematical model analyzed the immune response to a persistent, localized antigen (20). However, a single aggregated variable represented all the cytokines secreted by a cell type. Tumor's growth rate was estimated using a simple and abstract model of the immune system interactions with tumor cells (21). Mechanistic models of CD4+ T cells predicted novel phenotypes and response to different doses of cytokines $(6,15)$, including signaling pathways regulating differentiation. Furthermore, multi-scale models also considered both cell-cell communications and molecular signaling. Examples include a model predicting mucosal immune response to Helicobacter pylori infection (22), and a model predicting CD4+ T cell response to influenza infection (23). However, most of these works have either focused on limited aspects of the immune system (e.g., cell types) or modeled a single infection or a coinfection.

Here, we describe a comprehensive computational model of the human immune system and its interactions with bacteria, viruses, parasites, and fungi. The model includes all the components (cells and cytokines) of both innate and adaptive immune systems that are necessary to model responses to infections. The model was used to investigate the dynamics of the immune response under infection and coinfection of pathogens.

\section{Results}

We built a logical model of the immune system representing immune cells, cytokines, and immunoglobulins. We modeled the infections by nine pathogens using regulatory information of their interactions with target cells and immune cells obtained from the literature. The model was 
then used to investigate the dynamics of cytokines and immunoglobulins in response to monoand coinfections.

\section{Immune system model}

The model comprises 164 components representing 27 cell types and their various states, 36 molecules, and nine pathogens. To model the communication among these components, we manually curated 506 regulatory interactions from 220 peer-reviewed publications. We included pathogens from different groups: bacteria (Mycobacterium tuberculosis [MTB]), viruses (Influenza A virus [IAV], Human immunodeficiency virus [HIV], Human papillomavirus [HPV], Ebolavirus [EBOV]), parasites (Plasmodium falciparum [PF], Leishmania donovani [LD], Ascaris lumbricoides [AL]), and fungi (Candida albicans [CA]). The pathogens and their non-immune target cells (i.e., epithelial cells, erythrocytes) were included in the model as external inputs (i.e., stimuli) whose activity can be variable. Additionally, the variable "HealthyState" provides a global parameter to define the basal level of resting immune cells. The internal model components whose activities are dependent on external stimuli consist of: (1) resting immune cells provided by the external variable HealthyState, (2) uninfected target cells, (3) infected target cells, (4) activated immune cells, (5) secreted cytokines and immunoglobulins, (6) immune processes such as pathogen replication, vascular leakage. The interactions among components were modeled using logical functions consisting of the "AND," "OR," and "NOT" operators. Figure 1 A-C shows all the modeled pathogens, cells, and cytokines and immunoglobulins. Figure $1 \mathrm{D}$ shows the network diagram built from all regulatory interactions. The model can be accessed in the Cell Collective modeling platform (https://cellcollective.org) (24), and the SBML-qual (25) file is provided in Supplementary Data 1.

To validate the model, we first collected literature reporting specific immune response to modeled pathogens and simulated emergent behaviors that were not directly programmed into the model. As an example, we show validation results in the case of $M$. tuberculosis infection (Supplementary Table 1 provides all other validations). IFN- $\gamma$ was shown to be essential for resistance against MTB infection (26). The simulation results of our model showed that knock-out of IFN- $\gamma$ indeed increased the replication of $M$. tuberculosis (Figure $2 \mathrm{~A}$ ). We also investigated the activity of IL-6 during M. tuberculosis infection, as shown by Murray et al., 1999 (27). Similarly, we simulated a lower IL-6 in the case of IFN- $\gamma$ knock-out at high activity levels of $M$. tuberculosis (Figure 2 B). Additionally, similar to the patterns observed by Murray et al., 1999, IL-6 was increased at very high doses of the pathogen in the IL-10 knock-out (Supplementary Figure 1). In a double knock-out of IL-10 and IFN- $\gamma$, Murray et al., 1999 showed a higher IL-6 concentration, which was not replicated by our model (Supplementary Figure 2). However, as in Murray et al., the activity levels of IL-6 were higher in the simulated double knock-out compared to the simple IFN- $\gamma$ knock-out. Finally, IFN- $\gamma$ was shown to cause an increase in the number of CD8 cells during $M$. tuberculosis(28), a finding also replicated by 
our model at the high doses of the pathogen (Figure $2 \mathrm{C}$ ). The dose response curves for additional validations are shown in Supplementary Figure 3 - 7.
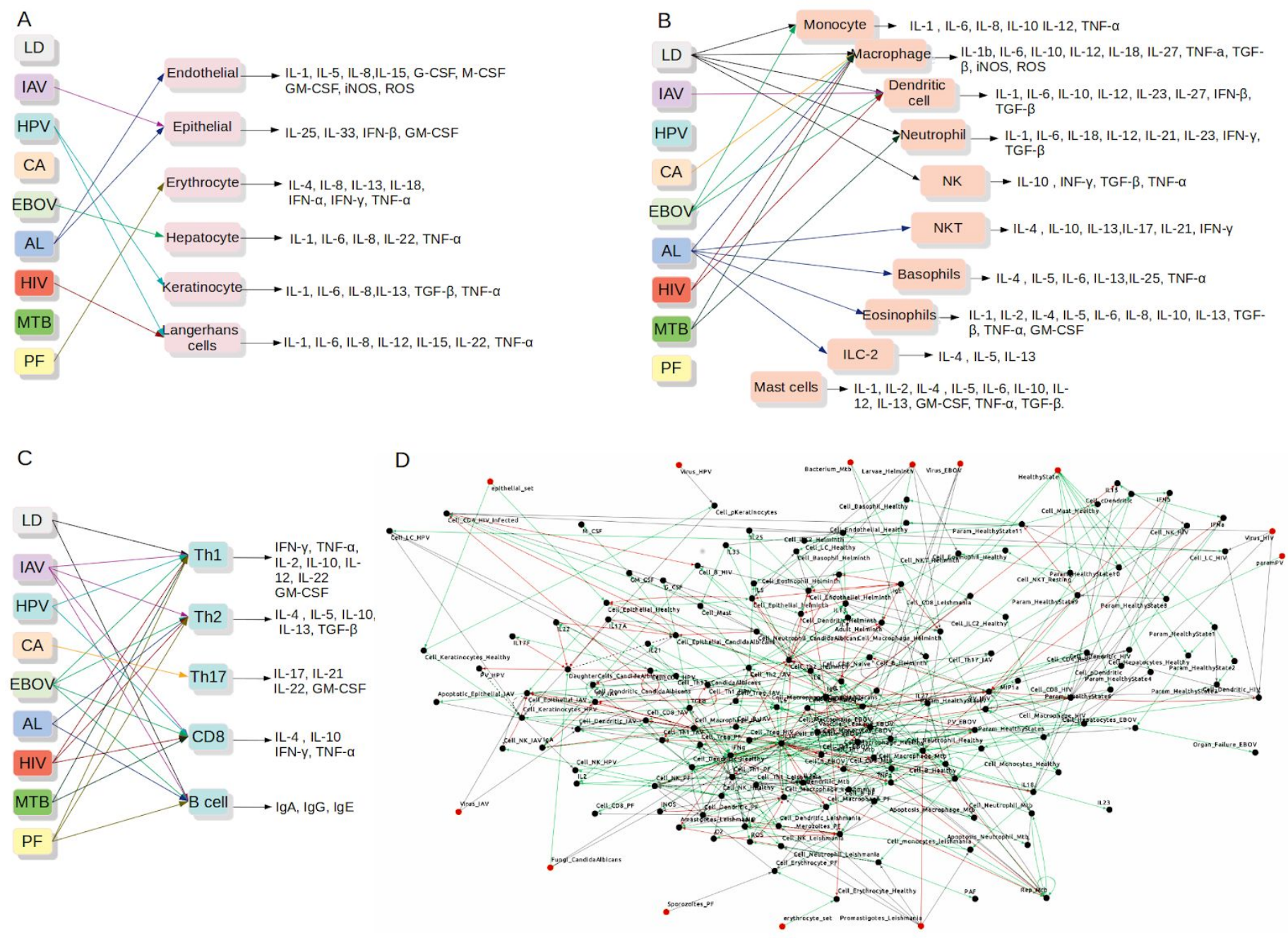

Figure 1: Components of the immune system model. (A) pathogens, non-immune target cells, and secreted cytokines (B) pathogens, innate immune target cells, and secreted cytokines, (C) pathogens, adaptive immune cells, and secreted cytokines and immunoglobulins. In panels A, B, C, the arrows connecting pathogens to cell types represent stimulation while the arrows connecting cell types and cytokines represent secretion (D) Network diagram of the model. Green arrows represent activation, red arrows represent inhibition, and gray arrows represent other conditions associated with activation and inhibitions (conditional activation and inhibition). Red nodes represent external (input) variables and black nodes represent internal components. 
A



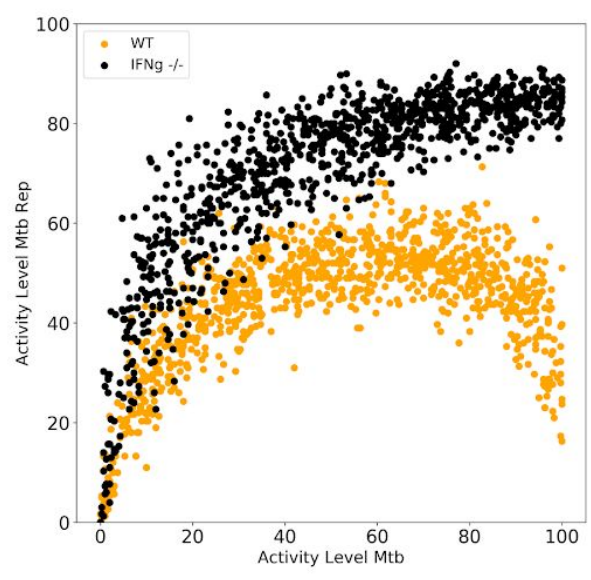

B

$\mathrm{IFN}-\mathrm{Y} \otimes \rightarrow \downarrow \mathrm{IL}-6$

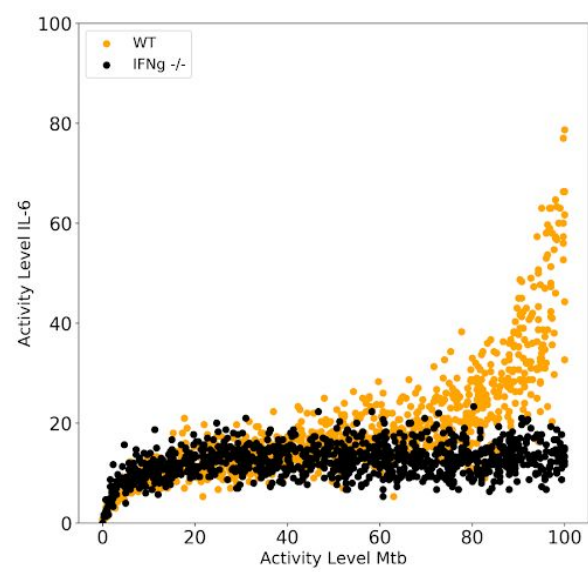

C
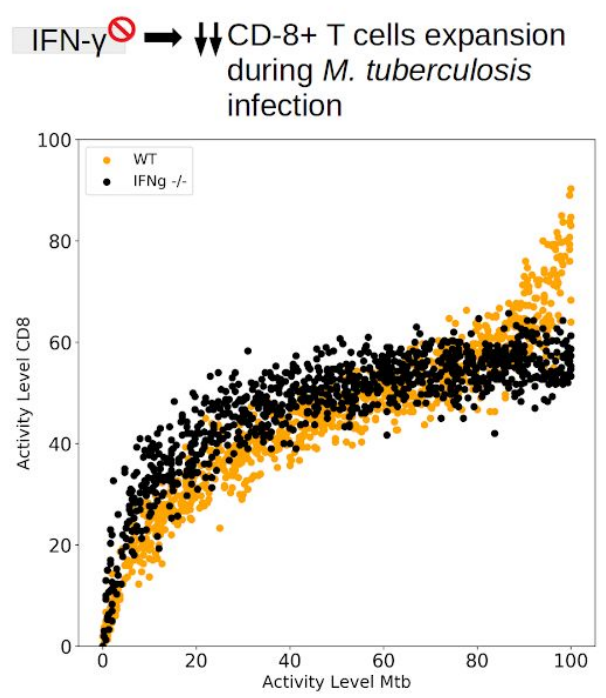

Figure 2: Model validation. Each panel shows the observation from literature after knock-outs of IFN- $\gamma$ and results of the model simulating the same behavior compared with wild type. (A) Activity levels of M. tuberculosis replication (Y-axis) in the presence (wild type; WT) and absence (IFN- $\gamma$--/--) of IFN- $\gamma$. (B) Activity levels of IL-6 (Y-axis) under WT, IFN- $\gamma$ knock-out conditions. (C) The activity of CD8 cells under WT and IFN- $\gamma$ knock-out conditions.

\section{Various infections trigger different cytokine responses}

We used model simulations to characterize the response of the immune system in the context of the cytokine and immunoglobulin activity levels under various infections (see Materials and Methods). We observed that the cytokine and immunoglobulin response to each infection varies (Figure $3 \mathrm{~A}$ ). EBOV infection stimulates a stronger response of both pro-inflammatory cytokines i.e., IL-1, IL-6, IL-12 and anti-inflammatory cytokines i.e., IL-8, and IL-10. HPV infection stimulates a strong response of anti-inflammatory cytokines IL-10 and TGF- $\beta$. AL infection results in a strong anti-inflammatory response in the form of IL-10, IL-4, IL-13, and IL-5. IAV infection stimulates robust pro-inflammatory response IL-1, IL-6, IL-12, IL-18, and TNF- $\alpha$. Cytokine response to PF is mixed pro- and anti-inflammatory, whereas MTB induces a strong pro-inflammatory cytokine response. Cytokine and immunoglobulin response to HIV is not significant because of the infection-driven, low activation of immune cells (e.g., CD4+ T cells).

Among all cytokines, IL-10, IFN- $\gamma$, and TNF- $\alpha$ were identified as the most active in response to simulated infection by different pathogens. Specifically, these cytokines were active in response to eight, seven, and five modeled pathogens, respectively (Figure $3 \mathrm{~A}$ ). 
IL-10 (Figure 3 B): Increased IL-10 response was observed in response to simulated infection by HPV, EBOV, AL, PF, IAV, and MTB. Among these infections, medium to high doses of HPV triggered the highest response of IL-10. Increased doses of EBOV corresponded to a linear increase of IL-10. We observed similar linear increases against AL and PF, but the IL-10 activity levels were lower than with EBOV. The activity of IL-10 decreased with increased doses of LD.

IFN- $\gamma$ : The activity level of IFN- $\gamma$ increased proportionally to the dose of MTB, PF, and LD. (Figure $3 \mathrm{C}$ ). Among these pathogens, MTB triggered the highest IFN- $\gamma$ response. Low to medium dosage of EBOV triggered high activities of IFN- $\gamma$, which subsequently decreased at a high dose. We observed a slight increase in IFN- $\gamma$ response against HPV and HIV and low IFN- $\gamma$ activity in response to high doses of CA.

TNF- $\alpha$ (Figure 3 D): The activity of TNF- $\alpha$ increased proportionally to the doses of EBOV, PF, IAV, and MTB, and initially increased and then decreased with increased doses of LD. We observed the highest TNF- $\alpha$ activity in response to EBOV infection.

A

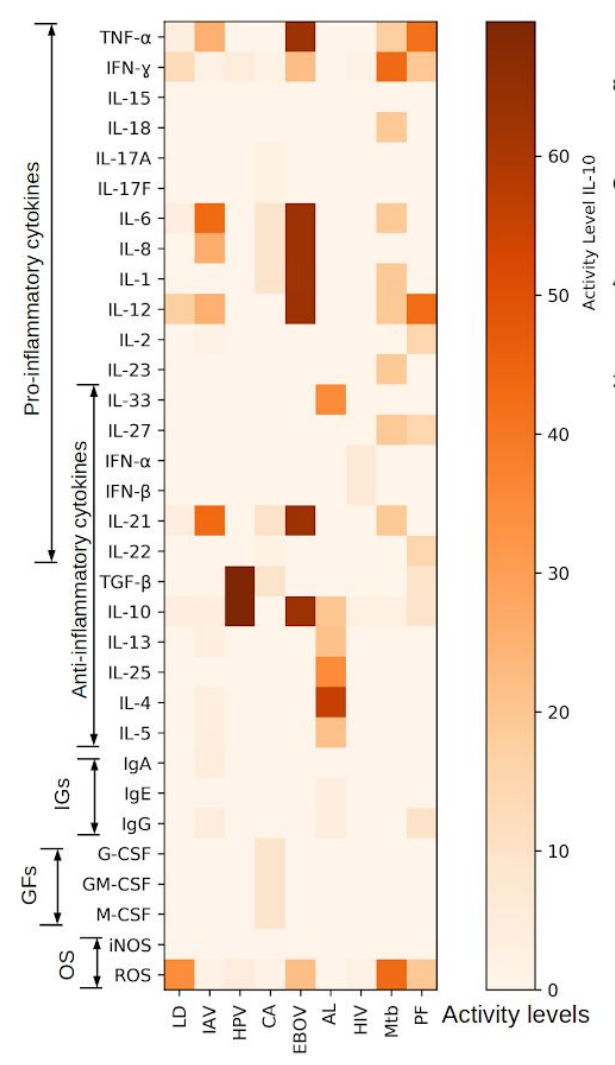

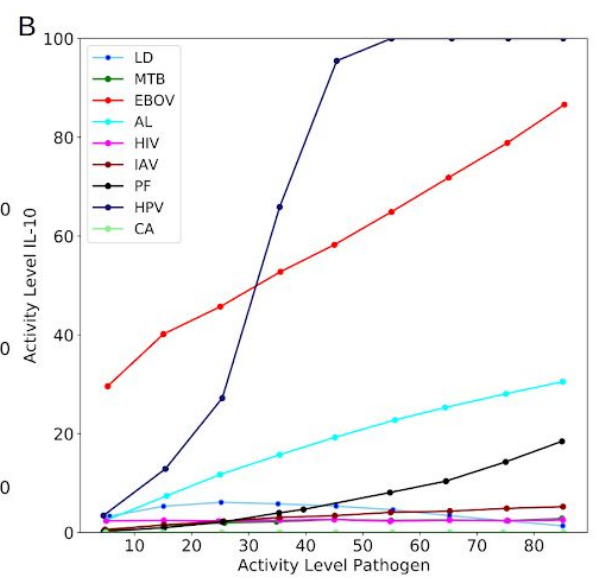
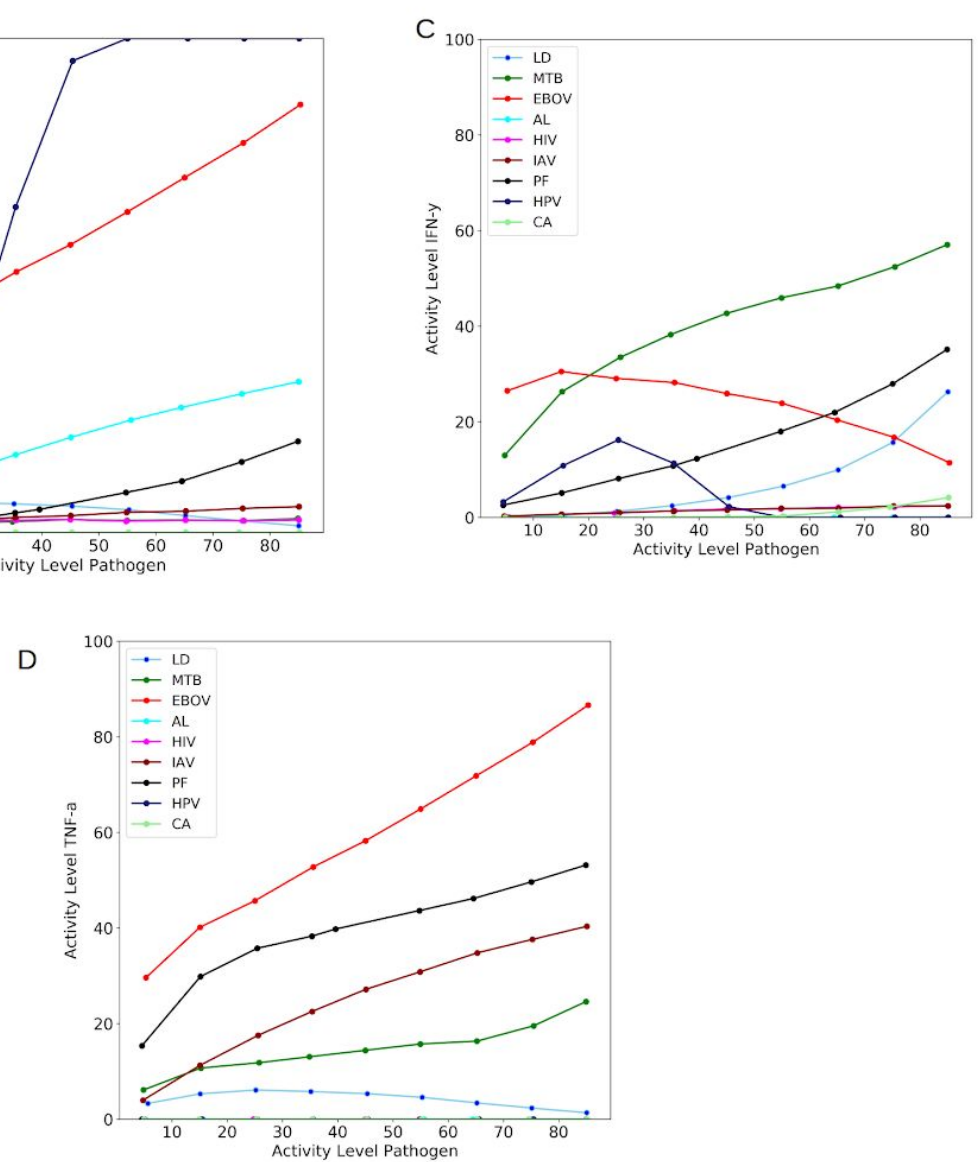

Figure 3: Immune response to infection with a single pathogen. (A) Activity levels of cytokines under infection with pathogens. Activity levels vary from $0 \%$ (no activity) to $100 \%$ 
(highest activity). The activity levels represent the average value triggered by pathogen levels from $0-100 \%$. IGs = immunoglobulins, GFs = growth factors, OS = oxidative stress. $(\mathrm{B}, \mathrm{C}$, and D) Dynamics of cytokines IL-10, IFN- $\gamma$, and TNF- $\alpha$ in response to different activity levels of pathogens.

\section{Response of specific cytokines to coinfections depends on the order of infections}

We studied the dynamics of the model in response to 17 medically relevant (observed) sequential coinfections (Supplementary Table 2; see Materials and Methods). For each coinfection, the order of infection was reversed and activity levels of cytokines and immunoglobulins were compared. Our results (Figure 4 A and 4 B) suggest that activity levels of cytokines and immunoglobulins are significantly different when the order of infections was reversed. Two types of analyses were performed.

First, we investigated which coinfection pairs displayed the largest number of differentially active immune molecules when the order of infection was reversed. We found that IAV-PF and HIV-CA coinfections were the most order-sensitive resulting in 21 (out of 32) modeled cytokines and immunoglobulins displaying significantly different activity levels. The AL-MTB, CA-MTB, and IAV-MTB coinfections resulted in 20 cytokines and immunoglobulins with differential activity levels. The order of infection was the least important in the MTB-LD and HIV-LD coinfections, where only 10 and 11 cytokines and immunoglobulins, respectively, displayed differential activities. The top two coinfections shared 13 differentially activated cytokines and immunoglobulins (IFN- $\gamma$, IL-1, IL-6, IL-8, IL-10, IL-12, IL-18, IL-21, IL-22, ROS, TGF- $\beta$, IgA, and IgG). The bottom two coinfections shared seven differentially activated cytokines and immunoglobulins (IFN- $\gamma$, IL-6, IL-10, IL-12, IL-21, ROS, and TNF- $\alpha$ ). The number of cytokines sensitive to the infection order in all studied coinfection pairs is shown in Figure 5 A.

Second, we investigated specific cytokines and immunoglobulins that were affected the most by the order of infection. IL-10 was strongly affected by the order of all pairs of infections. IFN- $\gamma$, IL-12, ROS, TNF- $\alpha$, IL-18, IL-21, IL-6, IFN- $\alpha$, IFN- $\beta$, IL-27, IL-23, IL-1, and TGF- $\beta$ also emerged as significantly affected. The activity levels of IFN- $\gamma$, IL-12, and ROS were significantly affected by the order of 16 out of 17 coinfections. TNF- $\alpha$ and IL-18 showed differential activity levels depending on the order of 15 coinfection pairs, while IL-21 activity affected by the order of 14 coinfection pairs. Among all investigated cytokines, only iNOS activity was not significantly affected by the order of any coinfections (Figure 5 B). 

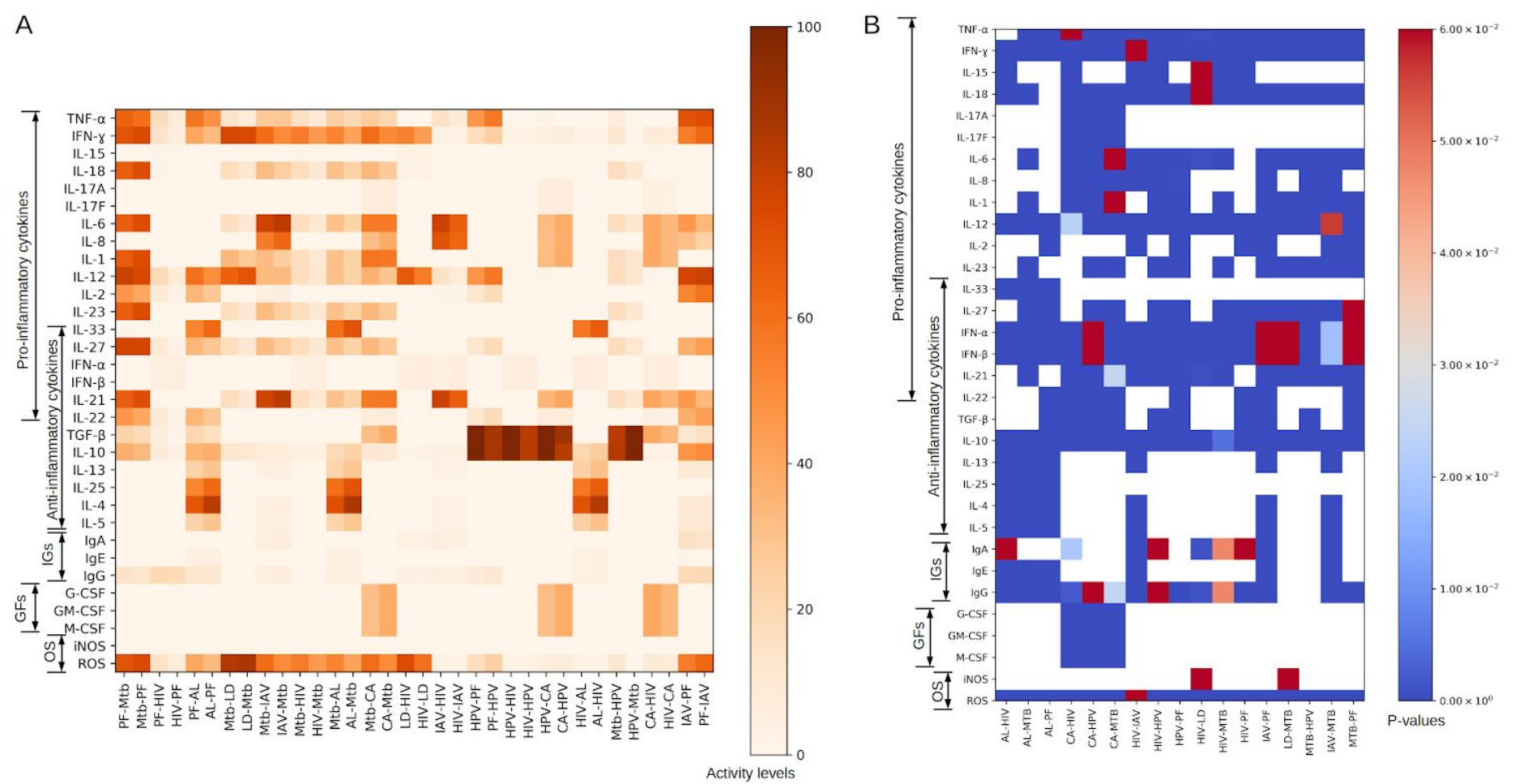

Figure 4: Immune response to coinfections. (A) Activity levels of cytokines in response to coinfection pairs under different orders. (B) P-values of compared activity profiles under a different order of infecting pathogens. White cells represent the inactivity of components in coinfection. IGs = immunoglobulins, GFs = growth factors, OS = oxidative stress. 
A
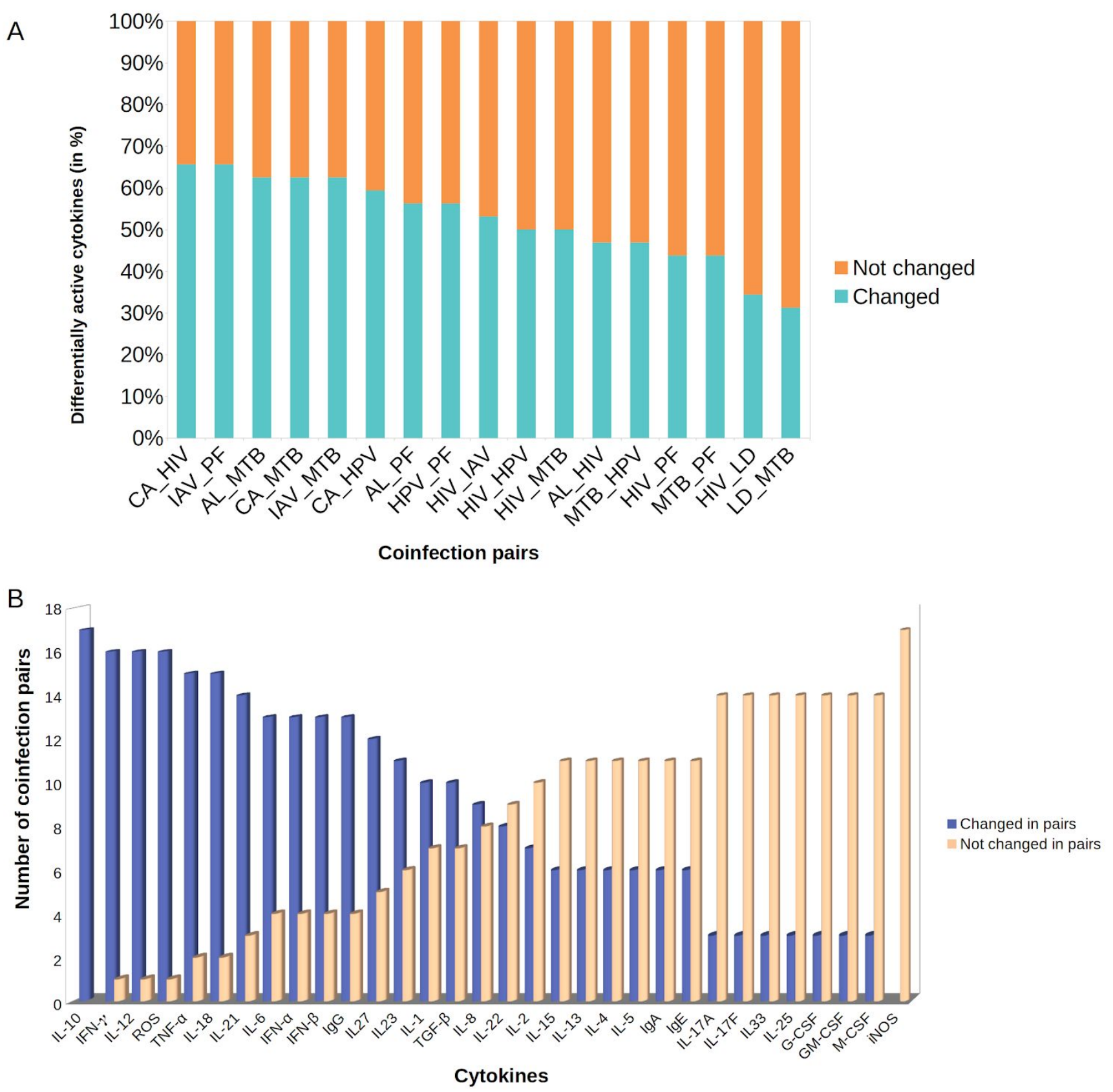

Figure 5: Differentially active cytokines under different coinfections: (A) \% of differentially active cytokines (out of 32 ) under different coinfections. Turquoise $=\%$ of 32 cytokines showed differential activity levels. Orange $=\%$ of 32 cytokines did not show differential activity levels. (B) Differentially active cytokines in $\%$ of coinfection pairs (out of 17 pairs). Blue= cytokines differentially activated in a number of 17 coinfections. Brown= cytokines not differentially activated in a number of 17 coinfections. 


\section{One pathogen is dominant in most coinfection pairs}

Next, to investigate which pathogen and what cytokines were dominant in the coinfections, we used hierarchical clustering and principal component analysis. We used activity levels of cytokines and immunoglobulins obtained under high infection strength (90-100\%) under different orders of coinfections. The clustering analysis of coinfections resulted in five clusters. Results (Figure $6 \mathrm{~A}$ ) show that different orders of the same coinfection have the lowest dissimilarity. Coinfections of HPV dominated Cluster 1 (Figure 6 A), composed of four coinfections. In cluster 2, AL was the dominant pathogen, whereas both MTB and LD were equally dominant in Cluster 3. Cluster 4 comprised two coinfections of PF. Cluster 5 was composed of four coinfections, two with MTB and two with HIV. Furthermore, we obtained seven clusters of cytokines using activity levels across different coinfections, in which TGF- $\beta$ and IL-10 were together with cluster A (Figure 6 B). All the immunoglobulins, growth factors, iNOS, were shown in cluster E (Figure $6 \mathrm{~B}$ ).

We performed the principal component analysis (PCA). Figure 6C and $6 \mathrm{D}$ show that $\mathrm{PC} 1$ and PC2 accounted for $57 \%$ of the variance in the data. Figure $6 \mathrm{C}$ shows a plot of the coinfections clusters along PC1, PC2, and PC3. Both PC1 and PC2 reflect high scores of coinfections in cluster 1 (i.e., dominated by HPV). PC1 is aligned with strong loadings of anti-inflammatory cytokines (TGF- $\beta$ and IL-10) versus pro-inflammatory loadings (IL-6, IL-12, IL-18, IFN- $\gamma$ ) (Figure 6C). PC2 is aligned with different groups of anti-inflammatory cytokines such as TGF- $\beta$ and IL-10 versus IL-4 and IL-25.

These results show that the HPV and AL were dominating other infections regardless of their order in the coinfection. The clustering of coinfections is influenced by the cytokines that showed variability in more than $50 \%$ of coinfections (discussed in the previous section, Figure $5)$, including IL-10, TGF- $\beta$, ROS, IFN- $\gamma$, IL-12, and TNF- $\alpha$. 



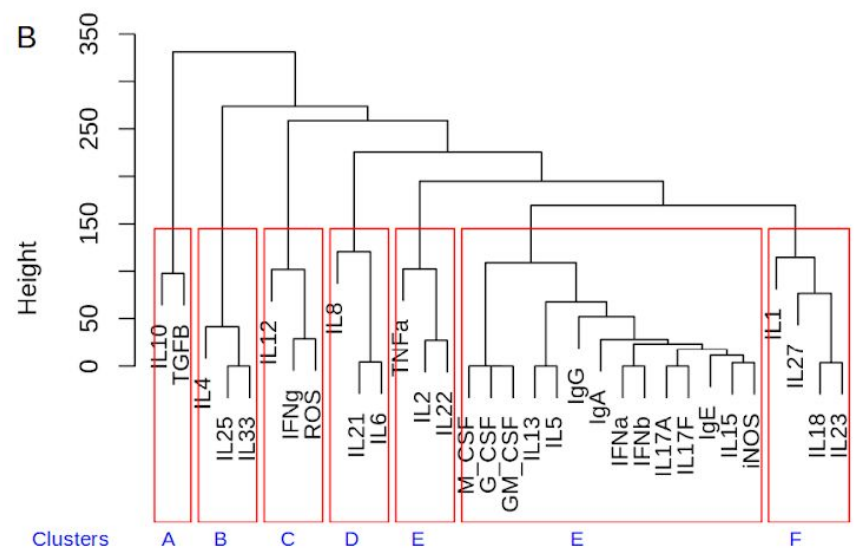

D



Figure 6: Clustering of coinfections and cytokines and immunoglobulins (A) Hierarchical clustering of coinfections, height $=$ distance (dissimilarity) between coinfections (B) hierarchical clustering of cytokines and immunoglobulins, height $=$ distance (dissimilarity) between components (C) Principal component analysis. Three-dimensional plot showing clusters of coinfections with PC1, PC2, and PC3. C1, C2, C3, C4, and C5 are clusters 1, 2,3, 4, and 5 identified in panel A. (D) Principal component analysis showing the main cytokine loadings upon PC1 and PC2.

\section{Discussion}

Computational models have become common tools to study the complexity of the immune system. However, most of these models focus on a specific part of the immune system. For instance, models have been developed to study differentiation of CD4+ T cells $(6,15,16)$, and the response to one $(19,29)$ or two pathogens $(30)$. Our model allows the interrogation of holistic 
immune system dynamics under nine pathogenic infections and/or any combination of coinfections.

The model's analysis under different infections showed varying cytokine and immunoglobulin responses. Dose-response analyses illustrated that most infections activate IL-10, IFN- $\gamma$, and TNF- $\alpha$, albeit at varying levels. In particular, HPV and EBOV resulted in the highest activity levels of IL-10, an important anti-inflammatory cytokine. A negative correlation between IL-10 and the pro-inflammatory cytokine IFN- $\gamma$ in HPV (Figure $3 \mathrm{~B}$ and C) suggests the stimulation of strong anti-inflammatory response. The higher activity of IL-10 during HPV infection is congruent with studies suggesting a role for IL-10 in immune evasion through immunosuppressive state (31). EBOV activated both anti- and pro-inflammatory cytokines at high levels. In addition to IL-10, our model suggested EBOV triggered a low activity of IFN- $\gamma$ and higher activities of other pro-inflammatory cytokines (TNF- $\alpha$, IL-1, IL-6, and IL-12, Figure $3 \mathrm{~A})$. These results confirm previous observations of a mix of both pro- and anti-inflammatory responses $(32,33)$.

Our results also suggest that the sequence of infections in a coinfection can significantly change the cytokine and immunoglobulin responses (Figure 4). Indeed, our model predicts that the many pro-inflammatory cytokines (e.g., IFN- $\gamma$, IL-12, TNF- $\alpha$, IL-18, and IL-21) and the anti-inflammatory cytokines (e.g., IL-10, IFN- $\alpha$, IFN- $\beta$ ), and ROS are differentially activated in more than $50 \%$ of cases when we reversed the order of infection. From published studies, it is clear that the order of infections is not only crucial in sequential infections, but also in co-transmitted infections, and super-infections. Furthermore, the order of infections can change dynamics and affect the prognosis (34). The importance of infection order has been discussed in the case of the parasite coinfections (35). Recently, it was shown that the sequence of larval trematodes in tadpoles could also affect the disease severity (36). In a different in vitro study in swine alveolar macrophages, the infection order of porcine circovirus type 2 and porcine reproductive and respiratory syndrome virus affected the cytokine response (37).

We used our model to predict the dominant pathogens in a coinfection, by comparing the activity profiles of cytokines and immunoglobulins across pathogens. Through clustering analysis, we identified one cluster of coinfections that was mostly made up of confections with HPV, regardless of the order. As we discussed previously, HPV exerts a robust anti-inflammatory response mediated by IL-10 and TGF- $\beta$, and creates an immunosuppressive environment. HPV infects primitive basal keratinocytes (38) that produce IL-10, which further facilitates the infection process via a positive feedback loop between IL-10 and HPV transcription(31). At the same time, HPV downregulates the expression of IFNs (39). Here we speculate that a strong anti-inflammatory response is the main reason behind HPV's dominance over other pathogens in coinfections. 
Finally, our model can identify cytokines that are affected by multiple coinfections. We found that IL-10, TGF- $\beta$, ROS, IFN- $\gamma$, IL-12, TNF- $\alpha$, IL-6, IFN- $\alpha$, and IFN- $\beta$ are the most affected cytokines. These observations are supported by a study on IAV with pneumococcal coinfection during which increased concentrations of IFN- $\gamma$, IL-6, and TNF- $\alpha$ were observed (40). Many coinfections affect IL-10. IAV-induced IL-10 secretion was found to help pneumococcal superinfection (41). Increased levels of IL-10 were observed in IAV-MTB coinfection. This was shown to compromise the host's ability to control the MTB infection (42). ROS was also identified as an essential molecule during the coinfection of human herpesvirus and chlamydia (43), further illustrating our model's predictive power.

Because the mechanism by which coinfections alter the immunity is unknown, computational models are useful to generate novel hypotheses. The model can be used as a tool (or a database) to understand the interactions among different immune cells and molecules under infection with modeled pathogens. As discussed above, by including more pathogens, one can investigate cytokines and immunoglobulin responses during mono and coinfection. By including drugs, the model can also be used to simulate the effects of drugs on pathogens. Finally, the model can be used for predictions related to other immune system-related pathologies. For example, a user could turn off all pathogens, and parametrize the model to study the dynamics under auto-immune disease conditions, immune response to transplants, etc.

\section{Materials and Methods}

\section{Model construction and mathematical framework}

The computational model is a logical boolean model, each component being either active (1) or inactive (0) at any given time $t$. The state of the components is updated based on the regulatory mechanism represented by Boolean functions (e.g., in the form of truth tables or Boolean expressions (44)). The regulatory mechanisms were defined using literature retrieved from PubMed (45). The model was constructed in Cell Collective (www.cellcollective.org) (24), a collaborative modeling platform for large-scale biological systems. To ensure transparency and reproducibility, each component and interaction in the model was annotated with information coming from the literature. The model is available for analysis in Cell Collective and can be retrieved for external analysis in the form of logical truth tables, as a list of Boolean expressions, in the Graph Markup Language (GML), and in the Systems Biology Markup Language (SBML) (46).

\section{Model simulation and analysis}

We performed the model simulations in Cell Collective (24). While the dynamical model is based on a discrete (i.e., Boolean) formalism, the output activity levels of individual components can be represented as semi-continuous values ranging from 0 to $100(24,47)$. The activity levels 
(dosage) of model stimuli are unit-less and defined as a per-cent chance (probability $* 100$ ) of the component being active in a given time $t$. Depending on the desired experiment, the user can set activity levels of external components to specific values, or ranges of activity levels (e.g., to simulate dose-response experiments). In the latter case, a probability (activity level) is selected at the beginning of a simulation and is used to set the input state to 0 or 1 at each time $t$. It is important to note that the activity levels provide a semi-quantitative measure of the relative activity of a particular component, instead of a specific biological measurement (e.g., concentration).

Each experiment simulating a single infection consisted of 1000 simulations with a different activity level randomly selected per simulation for the external components (in an activity range 0-100 \%). Each simulation was synchronous (i.e., all components are updated simultaneously) and lasted 800 steps. The activity levels of the output components were calculated as the fraction of 0 's and 1's over the last 300 iterations $(47,48)$. As noted previously (48) and from observations drawn from the model presented here (data not shown), the quick settling of the network makes these values sufficient to describe the network's "long-term" (e.g., attractor-like) behavior. For experiments simulating sequential coinfection, we adopted a three-stage approach (1) step 1 to step 99 were run without any infection, (2) at step 100, we added the first pathogen, and (3) at step 500, we added the second pathogen. We collected the data from steps 300 to 150. We ran one thousand simulations for each pair of infection. Perturbation analyses were performed in Cell Collective to evaluate which components' knockout or overexpression led to significant variations in the network dynamics. A knockout implies the deactivation of a component during an entire simulation, whereas an overexpression generates a persistent activation of a component.

\section{Simulation settings}

An input variable "HealthyState." The input node "HealthyState" was used as an analog to the steady state conditions of an uninfected cell. To simulate the immune response to different pathogens, first, we fixed the "HealthyState" input variable to $50 \%$, and then different doses of a pathogen are added to achieve the infected state. Dynamic simulations under these conditions activate downstream components in the model, and we retrieved that activity levels of internal components, including cytokines.

To represent coinfection, the input variable "HealthyState" was initially set to $50 \%$, and then different doses of two pathogens were added in the model one after the other (see above). Next, we reversed the order of pathogens. We executed this procedure for 17 coinfections reported in the literature (Supplementary Table 2). We then compared the activity levels of cytokines and immunoglobulins for the opposite sequences of infections. 


\section{Data analysis}

We performed the statistical, clustering, and principal component analysis in $\mathrm{R}$. The differential activities of cytokines and immunoglobulins were identified using Welch's two-sample T-test. Coinfections, cytokines, and immunoglobulins were clustered using the average hierarchical clustering (using hclust function) in R. The principal component analysis was performed using R's prcomp function. Python 3's MatPlotlib (49) was used to generate heatmaps and line plots.

\section{References}

1. Kaufmann SH. Immunity to intracellular bacteria. Annu Rev Immunol (1993) 11:129-163. doi:10.1146/annurev.iy.11.040193.001021

2. Braciale TJ, Hahn YS. Immunity to viruses. Immunol Rev (2013) 255: doi:10.1111/imr.12109

3. Anthony RM, Rutitzky LI, Urban JF, Stadecker MJ, Gause WC. Protective immune mechanisms in helminth infection. Nat Rev Immunol (2007) 7:975-987. doi:10.1038/nri2199

4. Richardson JP, Moyes DL. Adaptive immune responses to Candida albicans infection. Virulence (2015) 6:327-337. doi:10.1080/21505594.2015.1004977

5. Kaiko GE, Horvat JC, Beagley KW, Hansbro PM. Immunological decision-making: how does the immune system decide to mount a helper T-cell response? Immunology (2008) 123:326-338. doi:10.1111/j.1365-2567.2007.02719.x

6. Puniya BL, Todd RG, Mohammed A, Brown DM, Barberis M, Helikar T. A Mechanistic Computational Model Reveals That Plasticity of CD4+ T Cell Differentiation Is a Function of Cytokine Composition and Dosage. Front Physiol (2018) 9:878. doi:10.3389/fphys.2018.00878

7. Eizenberg-Magar I, Rimer J, Zaretsky I, Lara-Astiaso D, Reich-Zeliger S, Friedman N. Diverse continuum of CD4+ T-cell states is determined by hierarchical additive integration of cytokine signals. Proc Natl Acad Sci U S A (2017) 114:E6447-E6456. doi:10.1073/pnas.1615590114

8. Getahun H, Gunneberg C, Granich R, Nunn P. HIV infection-associated tuberculosis: the epidemiology and the response. Clin Infect Dis Off Publ Infect Dis Soc Am (2010) $\mathbf{5 0}$ Suppl 3:S201-207. doi:10.1086/651492

9. Park Y, Chin BS, Han SH, Yun Y, Kim YJ, Choi JY, Kim CO, Song YG, Kim JM. Pandemic Influenza (H1N1) and Mycobacterium tuberculosis Co-infection. Tuberc Respir Dis (2014) 76:84-87. doi:10.4046/trd.2014.76.2.84

10. Thompson MG, Breiman RF, Hamel MJ, Desai M, Emukule G, Khagayi S, Shay DK, Morales K, Kariuki S, Bigogo GM, et al. Influenza and malaria coinfection among young children in western Kenya, 2009-2011. J Infect Dis (2012) 206:1674-1684. doi:10.1093/infdis/jis591

11. Villani A-C, Sarkizova S, Hacohen N. Systems Immunology: Learning the Rules of the Immune System. Annu Rev Immunol (2018) 36:813-842. doi:10.1146/annurev-immunol-042617-053035

12. Yu J, Peng J, Chi H. Systems immunology: Integrating multi-omics data to infer regulatory networks and hidden drivers of immunity. Curr Opin Syst Biol (2019) 15:19-29. doi:10.1016/j.coisb.2019.03.003

13. Davis MM, Tato CM, Furman D. Systems immunology: just getting started. Nat Immunol (2017) 18:725-732. doi:10.1038/ni.3768

14. Saez-Rodriguez J, Simeoni L, Lindquist JA, Hemenway R, Bommhardt U, Arndt B, Haus U-U, Weismantel R, Gilles ED, Klamt S, et al. A Logical Model Provides Insights into T Cell Receptor Signaling. PLoS Comput Biol (2007) 3: doi:10.1371/journal.pcbi.0030163

15. Martinez-Sanchez ME, Huerta L, Alvarez-Buylla ER, Villarreal Luján C. Role of Cytokine Combinations on CD4+ T Cell Differentiation, Partial Polarization, and Plasticity: Continuous 
Network Modeling Approach. Front Physiol (2018) 9: doi:10.3389/fphys.2018.00877

16. Naldi A, Carneiro J, Chaouiya C, Thieffry D. Diversity and Plasticity of Th Cell Types Predicted from Regulatory Network Modelling. PLOS Comput Biol (2010) 6:e1000912. doi:10.1371/journal.pcbi.1000912

17. Puniya BL, Lichter B, Moore R, Ciurej A, Townsend S, Shah AR, Barberis M, Helikar T. Integrative computational approach identifies new targets in CD4+ T cell-mediated immune disorders. bioRxiv (2020)2020.01.02.893164. doi:10.1101/2020.01.02.893164

18. Bordbar A, Mo ML, Nakayasu ES, Schrimpe-Rutledge AC, Kim Y-M, Metz TO, Jones MB, Frank BC, Smith RD, Peterson SN, et al. Model-driven multi-omic data analysis elucidates metabolic immunomodulators of macrophage activation. Mol Syst Biol (2012) 8:558. doi:10.1038/msb.2012.21

19. Carbo A, Bassaganya-Riera J, Pedragosa M, Viladomiu M, Marathe $M$, Eubank S, Wendelsdorf K, Bisset K, Hoops S, Deng X, et al. Predictive Computational Modeling of the Mucosal Immune Responses during Helicobacter pylori Infection. PLOS ONE (2013) 8:e73365. doi:10.1371/journal.pone.0073365

20. Su B, Zhou W, Dorman KS, Jones DE. Mathematical Modelling of Immune Response in Tissues. Comput Math Methods Med (10) doi:https://doi.org/10.1080/17486700801982713

21. Sontag ED. A Dynamic Model of Immune Responses to Antigen Presentation Predicts Different Regions of Tumor or Pathogen Elimination. Cell Syst (2017) 4:231-241.e11. doi:10.1016/j.cels.2016.12.003

22. Verma M, Bassaganya-Riera J, Leber A, Tubau-Juni N, Hoops S, Abedi V, Chen X, Hontecillas R. High-resolution computational modeling of immune responses in the gut. GigaScience (2019) 8: doi:10.1093/gigascience/giz062

23. Wertheim KY, Puniya BL, Fleur AL, Shah AR, Barberis M, Helikar T. Multi-Approach and Multi-Scale Model of CD4+ T Cells Predicts Switch-Like and Oscillatory Emergent Behaviors in Inflammatory Response to Infection. bioRxiv (2020)2020.02.26.964502. doi:10.1101/2020.02.26.964502

24. Helikar T, Kowal B, McClenathan S, Bruckner M, Rowley T, Madrahimov A, Wicks B, Shrestha M, Limbu K, Rogers JA. The Cell Collective: Toward an open and collaborative approach to systems biology. BMC Syst Biol (2012) 6:96. doi:10.1186/1752-0509-6-96

25. Chaouiya C, Keating SM, Berenguier D, Naldi A, Thieffry D, lersel MP van, Le NN, Helikar T. SBML Level 3 package: Qualitative Models, Version 1, Release 1. J Integr Bioinforma (2015) 12:691-730. doi:10.1515/jib-2015-270

26. Flynn JL, Chan J, Triebold KJ, Dalton DK, Stewart TA, Bloom BR. An essential role for interferon gamma in resistance to Mycobacterium tuberculosis infection. J Exp Med (1993) 178:2249-2254. doi:10.1084/jem.178.6.2249

27. Murray PJ, Young RA. Increased Antimycobacterial Immunity in Interleukin-10-Deficient Mice. Infect Immun (1999) 67:3087-3095.

28. Booty MG, Nunes-Alves C, Carpenter SM, Jayaraman P, Behar SM. Multiple Inflammatory Cytokines Converge To Regulate CD8+ T Cell Expansion and Function during Tuberculosis. J Immunol (2016) 196:1822-1831. doi:10.4049/jimmunol.1502206

29. Obaid A, Naz A, Ikram A, Awan FM, Raza A, Ahmad J, Ali A. Model of the adaptive immune response system against HCV infection reveals potential immunomodulatory agents for combination therapy. Sci Rep (2018) 8:1-19. doi:10.1038/s41598-018-27163-0

30. Thakar J, Pilione M, Kirimanjeswara G, Harvill ET, Albert R. Modeling Systems-Level Regulation of Host Immune Responses. PLOS Comput Biol (2007) 3:e109. doi:10.1371/journal.pcbi.0030109

31. Berti FCB, Pereira APL, Cebinelli GCM, Trugilo KP, Brajão de Oliveira K. The role of interleukin 10 in human papilloma virus infection and progression to cervical carcinoma. Cytokine Growth Factor $\operatorname{Rev}(2017)$ 34:1-13. doi:10.1016/j.cytogfr.2017.03.002 
32. Banadyga L, Siragam V, Zhu W, He S, Cheng K, Qiu X. The Cytokine Response Profile of Ebola Virus Disease in a Large Cohort of Rhesus Macaques Treated With Monoclonal Antibodies. Open Forum Infect Dis (2019) 6: doi:10.1093/ofid/ofz046

33. Bixler SL, Goff AJ. The Role of Cytokines and Chemokines in Filovirus Infection. Viruses (2015) 7:5489-5507. doi:10.3390/v7102892

34. Metcalf CJE, Birger RB, Funk S, Kouyos RD, Lloyd-Smith JO, Jansen VAA. Five challenges in evolution and infectious diseases. Epidemics (2015) 10:40-44. doi:10.1016/j.epidem.2014.12.003

35. Karvonen A, Jokela J, Laine A-L. Importance of Sequence and Timing in Parasite Coinfections. Trends Parasitol (2019) 35:109-118. doi:10.1016/j.pt.2018.11.007

36. Pathirana NUK, Meegaskumbura M, Rajakaruna RS. Infection sequence alters disease severity-Effects of the sequential exposure of two larval trematodes to Polypedates cruciger tadpoles. Ecol Evol (2019) 9:6220-6230. doi:10.1002/ece3.5180

37. Tsai Y-C, Chang H-W, Jeng C-R, Lin T-L, Lin C-M, Wan C-H, Pang VF. The effect of infection order of porcine circovirus type 2 and porcine reproductive and respiratory syndrome virus on dually infected swine alveolar macrophages. BMC Vet Res (2012) 8:174. doi:10.1186/1746-6148-8-174

38. Stanley M. Immune responses to human papillomavirus. Vaccine (2006) 24:S16-S22. doi:10.1016/j.vaccine.2005.09.002

39. Torres-Poveda K, Bahena-Román M, Madrid-González C, Burguete-García Al, Bermúdez-Morales VH, Peralta-Zaragoza O, Madrid-Marina V. Role of IL-10 and TGF- $\beta 1$ in local immunosuppression in HPV-associated cervical neoplasia. World J Clin Oncol (2014) 5:753-763. doi:10.5306/wjco.v5.i4.753

40. Duvigneau S, Sharma-Chawla N, Boianelli A, Stegemann-Koniszewski S, Nguyen VK, Bruder D, Hernandez-Vargas EA. Hierarchical effects of pro-inflammatory cytokines on the post-influenza susceptibility to pneumococcal coinfection. Sci Rep (2016) 6:1-11. doi:10.1038/srep37045

41. Barthelemy A, Ivanov S, Fontaine J, Soulard D, Bouabe H, Paget C, Faveeuw C, Trottein F. Influenza A virus-induced release of interleukin-10 inhibits the anti-microbial activities of invariant natural killer T cells during invasive pneumococcal superinfection. Mucosal Immunol (2017) 10:460-469. doi:10.1038/mi.2016.49

42. Ring S, Eggers L, Behrends J, Wutkowski A, Schwudke D, Kröger A, Hierweger AM, Hölscher C, Gabriel G, Schneider BE. Blocking IL-10 receptor signaling ameliorates Mycobacterium tuberculosis infection during influenza-induced exacerbation. JCI Insight (2019) 4: doi:10.1172/jci.insight.126533

43. Prusty BK, Böhme L, Bergmann B, Siegl C, Krause E, Mehlitz A, Rudel T. Imbalanced Oxidative Stress Causes Chlamydial Persistence during Non-Productive Human Herpes Virus Co-Infection. PLOS ONE (2012) 7:e47427. doi:10.1371/journal.pone.0047427

44. Abou-Jaoudé W, Traynard P, Monteiro PT, Saez-Rodriguez J, Helikar T, Thieffry D, Chaouiya C. Logical Modeling and Dynamical Analysis of Cellular Networks. Front Genet (2016) 7: doi:10.3389/fgene.2016.00094

45. Sayers EW, Agarwala R, Bolton EE, Brister JR, Canese K, Clark K, Connor R, Fiorini N, Funk K, Hefferon T, et al. Database resources of the National Center for Biotechnology Information. Nucleic Acids Res (2019) 47:D23-D28. doi:10.1093/nar/gky1069

46. Chaouiya C, Bérenguier D, Keating SM, Naldi A, van lersel MP, Rodriguez N, Dräger A, Büchel F, Cokelaer T, Kowal B, et al. SBML qualitative models: a model representation format and infrastructure to foster interactions between qualitative modelling formalisms and tools. BMC Syst Biol (2013) 7:135. doi:10.1186/1752-0509-7-135

47. Helikar T, Rogers JA. ChemChains: a platform for simulation and analysis of biochemical networks aimed to laboratory scientists. BMC Syst Biol (2009) 3:58. doi:10.1186/1752-0509-3-58

48. Helikar T, Konvalina J, Heidel J, Rogers JA. Emergent decision-making in biological signal 
transduction networks. Proc Natl Acad Sci (2008) 105:1913-1918. doi:10.1073/pnas.0705088105

49. Hunter JD. Matplotlib: A 2D Graphics Environment. Comput Sci Eng (2007) 9:90-95.

doi:10.1109/MCSE.2007.55

\section{Author Contributions}

TH conceptualized the project. AM developed the immune system model, and BLP edited and finalized the model. BLP, RM, and RA simulated and analyzed the model dynamics. BLP, AM, and TH wrote the manuscript. All authors contributed to the manuscript.

\section{Funding}

This work has been supported by the National Institutes of Health grant \#1R35GM119770-04 to TH.

\section{Conflict of interest}

The authors declare that they have no conflict of interest. 